\title{
Hyalinizing Trabecular Tumor Masquerading as Papillary Thyroid Carcinoma on Fine-Needle Aspiration
}

\author{
Brittany E. Howard ${ }^{a}$ Sharon H. Gnagia ${ }^{a}$ I. Tolgay Ocal ${ }^{b}$ Michael L. Hinni ${ }^{a}$ \\ Departments of a Otolaryngology, Head and Neck Surgery and ${ }^{b}$ Pathology, Mayo Clinic \\ Arizona, Phoenix, Ariz., USA
}

\section{Key Words}

Hyalinizing trabecular adenoma - Fine-needle aspiration - Hyalinizing trabecular tumor .

Papillary thyroid carcinoma

\begin{abstract}
Background/Aims: Hyalinizing trabecular tumors are rare neoplasms of the thyroid gland often mistaken for thyroid carcinoma on fine-needle aspiration. We review the distinguishing characteristics of hyalinizing trabecular tumors and their management. Methods: We present the case of a patient diagnosed with papillary thyroid carcinoma on fine-needle aspiration as well as a review of the literature. Results: Intraoperative findings were felt to be inconsistent with papillary thyroid carcinoma and only a thyroid lobectomy was performed pending the results from pathologic evaluation, which confirmed a benign process and a diagnosis of hyalinizing trabecular tumor. Due to the diagnostic difficulty in distinguishing hyalinizing trabecular tumors from thyroid carcinomas on fine-needle aspiration, many patients receive overtreatment for what is primarily a benign disease. Conclusions: Recognition of hyalinizing trabecular tumors as a possible benign etiology of a thyroid mass can facilitate appropriate management.
\end{abstract}

(C) 2013 S. Karger AG, Basel

\section{Introduction}

Hyalinizing trabecular tumors are benign or low-malignant-potential tumors of the thyroid that were originally reported in 1905; however, they were not described in detail until 1987 by Carney et al. [1]. Due to its histologic features, the tumor is commonly

This report was a poster presentation at The Triological Society Combined Sections Meeting on January 24-26, 2013, in Scottsdale, Ariz., USA. 
Howard et al.: Hyalinizing Trabecular Tumor Masquerading as Papillary Thyroid Carcinoma on Fine-Needle Aspiration
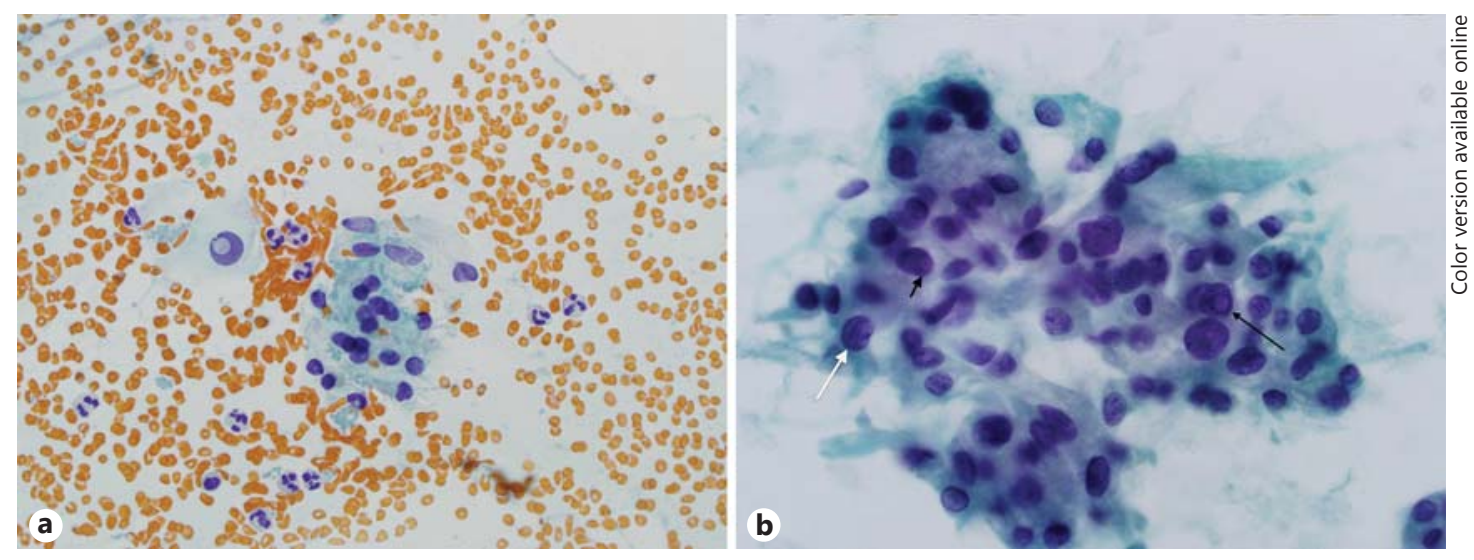

Fig. 1. a Scattered tumor cells in a bloody background with elongated nuclei and a well-formed nuclear pseudoinclusion. b Clustered cells with nuclear pseudoinclusions (long black arrow), perinuclear clearing (short black arrow), and nuclear grooves (white arrow).

Fig. 2. Gross specimen showing a soft tan-brown mass with a thick fibrous capsule that is characteristic of hyalinizing trabecular adenoma and atypical of papillary thyroid carcinoma.

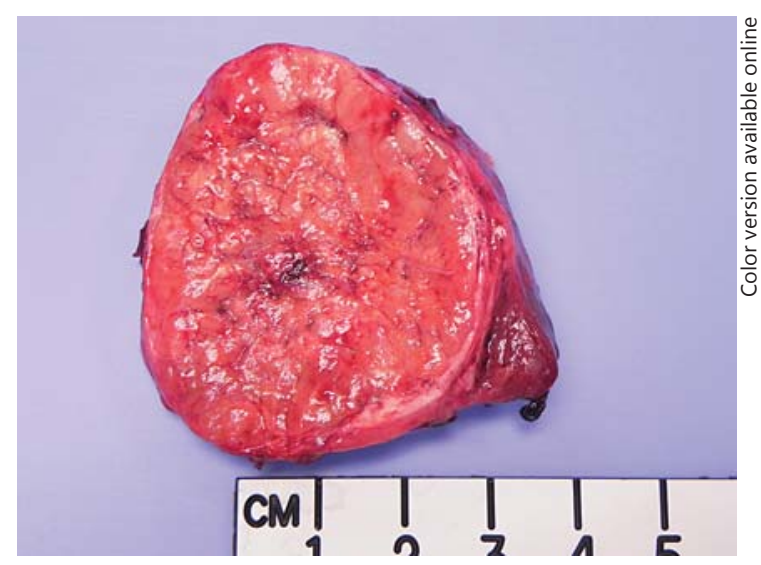

confused with thyroid carcinoma on fine-needle aspiration (FNA). This difficulty with the pathologic diagnosis often results in an overtreatment for what is almost universally a benign disease.

\section{Case Report}

A 57-year-old Native American female presented with a right-sided thyroid mass that, on CT scan, was found to be $6 \mathrm{~cm}$ in its greatest dimension. On imaging, the mass displayed substernal extension and caused significant tracheal deviation with a narrowing of the tracheal lumen to $50 \%$ of its normal caliber at the thoracic inlet. Although the mass had been present for over 10 years, it had recently undergone progressive growth and was causing symptoms of globus, dysphagia, and exertional shortness of breath. FNA showed cells with cytoplasmic invaginations, nuclear pseudoinclusions, and nuclear grooves (fig. 1). Based on these cytologic features, a diagnosis of papillary thyroid carcinoma was made and total thyroidectomy was planned.

Intraoperatively, a soft tan-brown mass with a thick fibrous capsule involving the right lobe of the thyroid was found (fig. 2). Its benign appearance raised suspicion for an etiology other than papillary thyroid carcinoma, although malignancy could not be excluded. A thyroid lobectomy was performed and frozen section analysis was requested prior to proceeding with total thyroidectomy. Frozen section analysis showed cells arranged in a trabecular pattern with extensive amounts of eosinophilic staining material, which was 
Howard et al.: Hyalinizing Trabecular Tumor Masquerading as Papillary Thyroid Carcinoma on Fine-Needle Aspiration
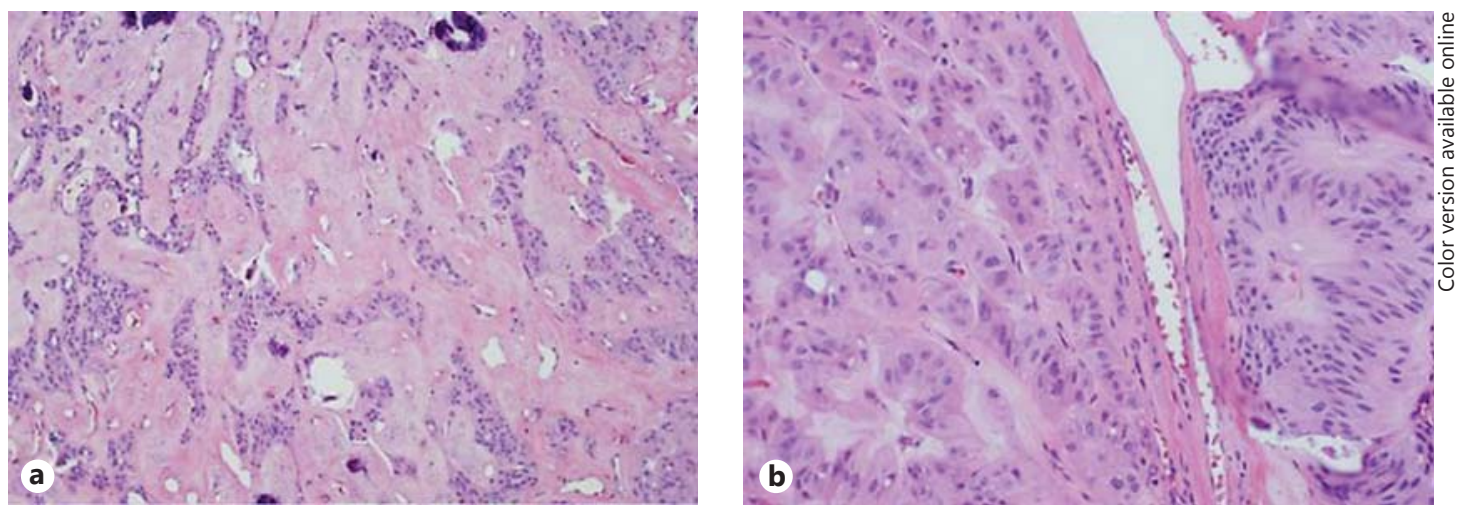

Fig. 3. a Eosinophilic staining-hyalinized areas are surrounded by cells in trabecular or nested arrangements. b Classical trabecular growth pattern of cells, which are oval or elongated in appearance. c Highpower view of cells showing typical nuclear findings of nuclear inclusions (black arrow) and perinucleolar clearing. Cytoplasmic yellow bodies (white arrow) are noted and represent lysosomes.

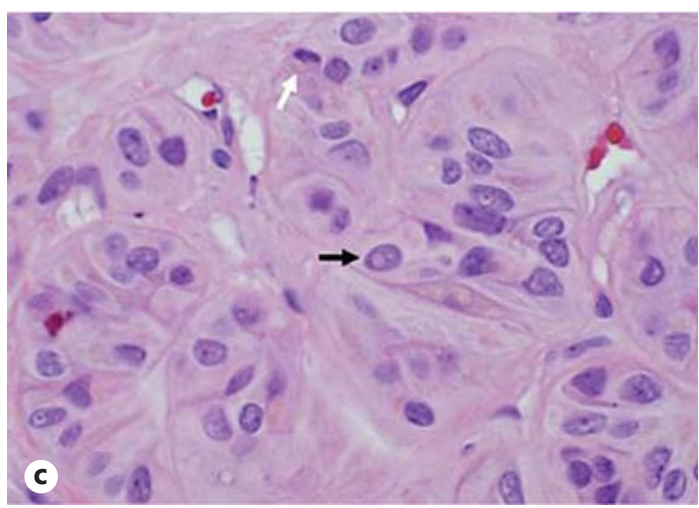

inconsistent with a diagnosis of papillary thyroid carcinoma. Based on these findings, total thyroidectomy was postponed pending the final pathology.

On pathology (fig. 3), the mass had a thick fibrous capsule without any evidence of capsular invasion. The cells were arranged in a trabecular pattern with an abundant pale eosinophilic cytoplasm. There was extensive hyalinization between the cellular trabeculae. Nuclear findings included perinucleolar clearing, nuclear pseudoinclusions, and nuclear grooves. Perinuclear yellow bodies were also identified. Calcitonin and polyclonal CEA immunostaining were negative, excluding the possibility of medullary thyroid carcinoma. A diagnosis of hyalinizing trabecular adenoma was made, and no further surgical intervention was recommended.

Following thyroid lobectomy, the patient experienced a significant improvement in her preoperative symptoms. She is euthyroid without thyroid replacement and has no evidence of recurrence at 6 months of follow-up.

\section{Discussion}

Hyalinizing trabecular tumors are rare tumors that are often mistaken for thyroid carcinomas on FNA. On FNA, both hyalinizing trabecular tumors and papillary thyroid carcinomas can demonstrate hypercellularity, psammoma bodies, and cellular atypia including cytoplasmic invaginations, nuclear grooves, and nuclear pseudoinclusions causing diagnostic difficulty. In the largest case series published so far, $81 \%$ of 55 patients undergoing FNA had lesions diagnosed as carcinoma or suspicious for carcinoma (diagnoses of papillary thyroid carcinoma or suspicious for papillary thyroid carcinoma accounted for $60 \%$ of all cases) [2]. Additionally, on FNA, the hyaline of hyalinizing trabecular tumors stains similar to amyloid, potentially causing misdiagnosis of medullary thyroid carcinoma. Findings on FNA that 
should raise the suspicion of hyalinizing trabecular tumor include a bloody background, cells with a low nuclear-to-cytoplasmic ratio, cellular aggregates around the hyaline material, fine chromatin (rather than the optically clear chromatin of papillary thyroid carcinoma), and numerous nuclear inclusions and grooves [3, 4].

On gross examination, hyalinizing trabecular tumors are usually well circumscribed or encapsulated. Their color typically ranges from yellow to tan, although variations have been described [2]. In comparison, papillary thyroid carcinomas are classically white and lack a capsule (extensive variation in their appearance has been described). Intraoperatively, an unexpected appearance in a thyroid mass may warrant further investigation, possibly including frozen section pathologic analysis. In our patient, a benign gross appearance combined with incongruent findings from the FNA and frozen section pathology prevented overtreatment.

The histologic appearance of hyalinizing trabecular tumors on frozen and permanent section has been described in exquisite detail by Carney and colleagues $[2,3,5]$ and is beyond the scope of this article. Briefly, the tumors have polygonal to elongated cells arranged in a trabecular or alveolar pattern. Hyaline material is present extensively in both intracellular and extracellular locations. The cell nuclei often have perinucleolar clearing, nuclear grooves, and nuclear inclusions similar to papillary thyroid carcinoma. Yellow cytoplasmic inclusions called 'yellow bodies' are a distinctive feature unique from papillary thyroid carcinomas and represent lysosomes. Calcifications and psammoma bodies can be seen interspersed throughout the stroma.

There has been significant debate on the most appropriate terminology for these tumors since their original description as hyalinizing trabecular adenomas by Carney et al. [5] in 1987. Due to the concern of a possible relationship with papillary thyroid carcinoma and case reports of malignant variants, the term 'hyalinizing trabecular tumors' was proposed and is currently the classification accepted by the World Health Organization. The possibility that hyalinizing trabecular tumors represent a variant of papillary thyroid carcinoma has been considered given their similar cytologic findings, RET/PTC rearrangements in some tumors [6], and variable expression of the galectin-3 molecular marker [7]. Cheung et al. [8] have previously reported that the RET/PTC gene rearrangements found in hyalinizing trabecular tumors confirmed the 'long-standing suspicion' that these tumors are in fact a variant of papillary thyroid carcinoma. However, on further genetic analysis, hyalinizing trabecular tumors have since been shown to be a discrete entity from papillary thyroid carcinoma with an absence of BRAF mutations [6], differential expression of miRNAs [9], and unique staining patterns for molecular markers including CD56 and MIB-1 [10,11].

Hyalinizing trabecular tumors have an exceedingly low to nonexistent malignant potential. Although malignant variants have previously been described in case reports, a review of the cases raises the possibility of misdiagnosis of thyroid carcinoma with a focal hyalinized or trabecular growth pattern [12]. In the largest case series by Carney et al. [2] including 119 cases, 118 were benign and found to meet the strict diagnostic criteria for hyalinizing trabecular adenomas. The single malignant tumor was classified as a hyalinizing trabecular carcinoma and was clinically distinct. This tumor did not meet the diagnostic criteria for an adenoma due to evidence of capsular invasion, numerous mitoses, and pulmonary metastasis at presentation. The 118 tumors categorized as hyalinizing trabecular adenomas behaved benignly without recurrence or metastases with up to 48 years of followup. Therefore, those tumors meeting the diagnostic criteria for hyalinizing trabecular tumors can be viewed as benign or at least of minimal malignant potential.

Given the primarily benign nature of hyalinizing trabecular tumors, they are appropriately and adequately treated with thyroid lobectomy alone. Due to the difficulty in distinguishing these tumors from thyroid carcinomas on FNA, the literature shows that $44-71 \%$ of 
patients receive overtreatment with total or subtotal thyroidectomy [2, 4]. For this reason, it is important that clinicians are aware of hyalinizing trabecular tumors as a benign etiology of thyroid masses masquerading as thyroid carcinomas.

\section{Conclusion}

Hyalinizing trabecular tumors are rare tumors of the thyroid gland typified by a trabecular or alveolar architecture with extensive hyaline and colloid deposits. Their cellular features of cytoplasmic inclusions, nuclear pseudoinclusions, nuclear grooves, and psammoma bodies often cause them to be mistaken for papillary thyroid carcinomas on FNA. They represent benign or low-malignant-potential tumors that are adequately treated by thyroid lobectomy. An awareness of hyalinizing trabecular tumors and their characteristic features is valuable for their recognition and management as well as for the possible prevention of overtreatment for benign disease.

\section{Disclosure Statement}

The authors have no financial disclosures related to this research. We do wish to disclose that Dr. Hinni's employer owns the rights to his invention of a laryngoscope and that his employer has licensed it to Karl Storz for potential manufacturing.

\section{References}

$\checkmark 1$ Carney JA: Hyalinizing trabecular tumors of the thyroid gland: quadruply described but not by the discoverer. Am J Surg Pathol 2008;32:622-634.

2 Carney JA, Hirokawa M, Lloyd RV, Papottie M, Sebo TJ: Hyalinizing trabecular tumors of the thyroid gland are almost all benign. Am J Surg Pathol 2008;32:1877-1889.

3 Casey MB, Thomas JS, Carney JA: Hyalinizing trabecular adenoma of the thyroid gland: cytologic features in 29 cases. Am J Surg Pathol 2004;28:859-867.

4 Kim T, Oh YL, Shin YH: Diagnostic dilemmas of hyalinizing trabecular tumors on fine needle aspiration cytology: a study of seven cases with BRAF mutation analysis. Cytopathology 2011;22:407-413.

5 Carney JA, Ryan J, Goellner JR: Hyalinizing trabecular adenoma of the thyroid gland. Am J Surg Pathol 1987; 11:583-591.

- 6 Salvatore G, Chiappetta G, Nikiforov YE, Decaussin-Petrucci M, Fusco A, Carney JA, Santoro M: Molecular profile of hyalinizing trabecular tumours of the thyroid: high prevalence of RET/PTC rearrangements and absence of B-raf and N-ras point mutations. Eur J Cancer 2005;41:816-821.

7 Gaffney RL, Carney JA, Sebo TJ, Erickson LA, Volante M, Papotti M, Lloyd RV: Galectin-3 expression in hyalinizing trabecular tumors of the thyroid gland. Am J Surg Pathol 2003;27:494-498.

- 8 Cheung CC, Boerner SL, MacMillan CM, Ramyar L, Asa SL: Hyalinizing trabecular tumor of the thyroid: a variant of papillary carcinoma proved by molecular genetics. Am J Surg Pathol 2000;24:1622-1626.

9 Sheu S, Vogel E, Worm K, Grabellus F, Schwertheim S, Schmid KW: Hyalinizing trabecular tumor of the thyroid - differential expression of distinct miRNAs compared with papillary thyroid carcinoma. Histopathology 2010;56:632-640.

10 Lee S, Hong S, Koo JS: Immunohistochemical subclassification of thyroid tumors with prominent hyalinizing trabecular pattern. APMIS 2011;119:529-536.

11 Casey MB, Sebo TJ, Carney JA: Hyalinizing trabecular adenoma of the thyroid gland identification through MIB-1 staining of fine-needle aspiration biopsy smears. Am J Clin Pathol 2004;122:506-510.

12 Galgano MT, Mills SE, Stelow EB: Hyalinizing trabecular adenoma of the thyroid revisited: a histologic and immunohistochemical study of thyroid lesions with prominent trabecular architecture and sclerosis. Am J Surg Pathol 2006;30:1269-1273. 